\title{
ON ORDERS SOLELY OF ABELIAN GROUPS
}

\section{by S. SRINIVASAN}

(Received 6 November, 1985)

1. Introduction. Let $n=\prod_{i=1}^{r} p_{i}^{a_{i}}$ be the factorization of an integer $n(>1)$ into prime powers, and set $\Phi(n):=\prod_{i=1}^{r}\left(p_{i}^{a_{i}}-1\right)$. In particular, for squarefree $n, \Phi(n)=\phi(n)$. Consider the set

$$
A:=\left\{n: 1 \leqslant a_{i} \leqslant 2,1 \leqslant i \leqslant r ;(n, \Phi(n))=1\right\} .
$$

It is known (from [5]) that $A$ consists precisely of those integers $n$ for which there is no non-abelian group of order $n$. It is also known (from [7]) that the set

$$
C:=\{n: n \in A, n \text { squarefree }\}
$$

consists solely of integers $n$ with the property that every group of order $n$ is cyclic. We set $C^{\prime}=A-C$.

For a sequence $B$ of integers, let $B(x)$ denote the number of $m \in B$ with $m \leqslant x$. In [1], Erdös proved that

$$
C(x) \sim e^{-\gamma} x L_{3}^{-1}, \quad(x \rightarrow \infty)
$$

in the notation $L_{1}:=\log x, L_{r+1}:=\log L_{r}(r \geqslant 1)$, where $\gamma$ is Euler's constant. Recently, in [8], Warlimont considered $C^{\prime}(x)$ and showed that

$$
x L_{2}^{-1} L_{3}^{-2} \ll C^{\prime}(x) \ll_{\varepsilon} x L_{2}^{-1} L_{3}^{\epsilon-1 / 2}, \quad(x \rightarrow \infty)
$$

for every $\varepsilon>0$. In the present paper we show that here one can also have the lower estimate as the upper bound. Thus we obtain the following theorem.

THEOREM. We have

$$
C^{\prime}(x) \breve{\curvearrowleft} x L_{2}^{-1} L_{3}^{-2},
$$

as $x \rightarrow \infty$.

Remark. The proof here uses a result from the large sieve instead of the result from [2] which was employed in [8] in obtaining the upper bound in (2).

2. Some lemmas. The following lemma, derived from the large sieve, is basic in the proof.

Lemma 1. Let $q(m)$ denote the least prime divisor of $m$ and write

$$
S(x, y, p):=\sum_{\substack{m \leq x \\(p, \phi(m))=1 \\ q(m) \geqslant y}} 1 .
$$

Glasgow Math. J. 29 (1987) 105-108. 
Then, for $(2 \leqslant) y \leqslant p \leqslant(\log x)^{1 / 4}$, we have

$$
S(x, y, p) \leqslant c_{0} \frac{x}{\log y} \exp \left(-\frac{\log \log x}{10 p}\right), \quad x \rightarrow \infty
$$

where $c_{0}$ is an absolute constant.

Proof. In Theorem 7.1 of [6] (which is practically the Corollary in [4]), take $N=x$, $z=x^{1 / 2}$ (say) and, for primes $q, \omega(q)=1$ if either $q \leqslant y$ or $q \equiv 1(\bmod p)$ and $\omega(q)=0$ otherwise. This gives

where

$$
S(x, y, p) \leqslant \frac{2 x}{L(z)}
$$

$$
\dot{L}(z):=\sum_{m \leqslant z} \mu^{2}(m) \prod_{q \mid m} \frac{\omega(q)}{q-\omega(q)}
$$

Now from (9.38) of [6], since $\omega(q)=0$ or 1 , it follows that

$$
L(z) \geqslant \prod_{\substack{q \leqslant z \\ \omega(q)=0}}\left(1-\frac{1}{q}\right) \log z .
$$

On using $\log z \geqslant \frac{2}{3} \prod_{q \leqslant z}\left(1-\frac{1}{q}\right)^{-1}$ (say, for large $z$ ), we obtain, from the above estimates,

$$
S(x, y, p) \leqslant 3 x \prod_{q \leqslant x^{12}}\left(1-\frac{\omega(q)}{q}\right) .
$$

This bound yields the result of Lemma 1, in view of the definition of $\omega(q)$ and the prime number theorem for the arithmetic progression of integers congruent to $1 \bmod p$.

Remark. Here the condition $p \leqslant(\log x)^{1 / 4}$ is imposed only for making $c_{0}$ effective.

For convenience of reference we state the next simple lemma. However, for our present purpose, we only need the upper bound given by this lemma.

LEMMA 2. We have

as $X / Y \rightarrow \infty$.

$$
\sum_{p>Y} \frac{\log p}{p^{2}} \exp (-X / p) \sim X^{-1}
$$

Proof. Writing $\theta(u):=\sum_{p \leqslant u} \log p$ and $b(u)=u^{-2} \exp (-X / u)$, we have

$$
\sum_{p>Y} \frac{\log p}{p^{2}} \exp (-X / p)=\sum_{m>Y} \theta(m)(b(m)-b(m+1))+O(\theta(Y+1) b(Y+1)) .
$$

Using $\theta(u) \sim u, u \rightarrow \infty$ (cf. for example [3, Theorem 434, p. 362]) we see that the above 
quantity equals

$$
\sum_{m>Y} m(b(m)-b(m+1))+O\left(Y^{-1} \exp (-X / Y)\right)+o\left(X^{-1}\right)
$$

since $b(u)$ is monotonic in $\left(Y, \frac{1}{2} X\right)$ and $\left(\frac{1}{2} \mathrm{X}, \infty\right)$. Now, as $X / Y \rightarrow \infty$, the last expression equals $\sum_{m>Y} b(m)+o\left(X^{-1}\right) \sim X^{-1}$. This proves the lemma.

3. Proof of the theorem. To start with, we have

$$
C^{\prime}(x) \leqslant \sum_{1<k \leqslant x} \sum_{\substack{m \leqslant x k^{-2} \\ m k \in C}} 1 \leqslant \sum_{1<k \leqslant Z} \sum_{\substack{m \leqslant x k^{-2} \\ m k \in C}} 1+O\left(x Z^{-1}\right)
$$

for any $Z \leqslant x$. Now let $Y \leqslant Z$ be another parameter to be chosen later. In the last double summation of (4) we consider those $m k$ having a prime divisor $q \leqslant Y$. For each prime $q \leqslant Y$, the number of such $m k(\leqslant x)$ having $q$ for the least prime divisor does not exceed, by Lemma 1 (with $p=q, y=2$, say),

$$
c_{0}(\log 2)^{-1} x \exp \left(-L_{2} / 10 q\right)
$$

since $m k \in C$. Hence the number of $m k^{2}$ under consideration in (4) is

$$
O\left(x Z \sum_{q \leqslant Y} \exp \left(-L_{2} / 10 q\right)\right)=O\left(x Z^{2} \exp \left(-L_{2} / 10 Y\right)\right)
$$

Choosing here $Y=L_{2}^{3 / 4}=Z^{1 / 2}$, say, it follows from (4) that

$$
C^{\prime}(x) \leqslant \sum_{Y<k \leqslant Y^{2}} \sum_{m}^{*} 1+O\left(x L_{2}^{-3 / 2}\right)
$$

with ${ }^{*}$ signifying the restrictions (i) $m \leqslant x k^{-2}$, (ii) $m k \in C$ and (iii) the least prime divisor of $m k$ exceeds $Y$. Now, these conditions imply that $k$ is a prime ( $p$, say). Again, by Lemma 1 (with $y=Y$ and $x p^{-2}$ for $x$ ) we obtain

$$
\begin{aligned}
\sum_{Y<p \leqslant Y^{2}} \sum_{m}^{*} 1 & =\sum_{Y<p \leqslant Y^{2}} S\left(x / p^{2}, Y, p\right) \\
& \ll \sum_{Y<p \leqslant Y^{2}} \frac{x \log p}{p^{2}(\log Y)^{2}} \exp \left(-\frac{\log \log x}{10 p}\right) \\
& \ll \frac{x}{(\log Y)^{2}} \sum_{p>Y} \frac{\log p}{p^{2}} \exp \left(-\frac{\log \log x}{10 p}\right) .
\end{aligned}
$$

Therefore, by our choice of $Y$ and Lemma 2 (with $X=L_{2} / 10$, noting that $X / Y \rightarrow \infty$ ), we conclude from $\left(4^{\prime}\right)$ that

$$
C^{\prime}(x)=O\left(x L_{3}^{-2} L_{2}^{-1}+x L_{2}^{-3 / 2}\right) .
$$

Combining the lower estimate in (2) with (5) completes the proof of the theorem. 
ACKNOWLEDGEMENT. The author wishes to thank the referee for some helpful suggestions.

\section{REFERENCES}

1. P. Erdös, Some asymptotic formulas in number theory, J. Indian Math. Soc. (N.S.) 12 (1948), 75-78.

2. R. R. Hall, Halving an estimate obtained from Selberg's upper bound method, Acta Arith. 25 (1973/4), 347-351.

3. G. H. Hardy and E. M. Wright, Introduction to the theory of numbers, 4th edn. (Oxford University Press, 1964).

4. H. L. Montgomery, A note on the large sieve, J. London Math. Soc. 43 (1968), 93-98. 225-264.

5. L. Rédei, Das "schiefe Produkt" in der Gruppen theorie, Comment. Math. Helv. 20 (1947),

6. H.-E. Richert, Sieve methods (Tata Institute Lecture Notes, Bombay, 1976).

7. T. Szele, Uber die endlichen Ordnungszahlen, zu denen nur eine Gruppe gehört, Comment. Math. Helv. 20 (1947), 265-267.

8. R. Warlimont, On the set of natural numbers which only yield orders of abelian groups, $J$. Number Theory 20 (1985), 354-362.

School of Mathematics

TATa Institute of Fundamental Research

HOMI BHaBHa ROAD

BOMBAY 400005

INDIA 